\title{
The transplantation of Akt-overexpressing amniotic fluid-derived mesenchymal stem cells protects the heart against ischemia-reperfusion injury in rabbits
}

\author{
YAN WANG ${ }^{1}$, YIGANG LI ${ }^{2}$, LEI SONG ${ }^{2}$, YANYAN LI ${ }^{2}$, SHAN JIANG ${ }^{2}$ and SONG ZHANG ${ }^{2}$ \\ Departments of ${ }^{1}$ Geriatrics and ${ }^{2}$ Cardiovascular Diseases, Xinhua Hospital, \\ School of Medicine, Shanghai Jiaotong University, Shanghai 200092, P.R. China
}

Received July 31, 2015; Accepted April 22, 2016

DOI: $10.3892 / \mathrm{mmr} .2016 .5212$

\begin{abstract}
Amniotic fluid-derived mesenchymal stem cells (AFMSCs) are an attractive cell source for applications in regenerative medicine, due to characteristics such as proliferative capacity and multipotency. In addition, Akt, a serine-threonine kinase, maintains stem cells by promoting viability and proliferation. Whether the transplantation of Akt-overexpressing AFMSCs protects the heart against ischemia-reperfusion (I/R) injury has yet to be elucidated. Accordingly, the Akt gene was overexpressed in AFMSCs using lentiviral transduction, and Akt-AFMSCs were transplanted into the ischemic myocardium of rabbits prior to reperfusion. Any protective effects resulting from this procedure were subsequently sought after three weeks later. A histological examination revealed that there was a decrease in intramyocardial inflammation and ultrastructural damage, and an increase in capillary density and in the levels of GATA binding protein 4, connexin 43 and cardiac troponin $\mathrm{T}$ in the Akt-AFMSC group compared with the control group. A significant decrease in cardiomyocyte apoptosis, accompanying an increase in phosphorylated Akt and B-cell lymphoma 2 (Bcl-2) and a decrease in caspase-3, was also observed. Furthermore, the left ventricular function was markedly augmented in the Akt-AFMSC group compared with the control group. These observations suggested that the protective effect of AFMSCs may be due to the delivery of secreted cytokines, promotion of neoangiogenesis, prevention of cardiomyocyte apoptosis, transdifferentiation into cardiomyocytes and promotion of the viability of AFMSCs, which are assisted by Akt gene modification. Taken together, the results of the present study have indicated that transplantation
\end{abstract}

Correspondence to: Dr Song Zhang, Department of Cardiovascular Diseases, Xinhua Hospital, School of Medicine, Shanghai Jiaotong University, 1665 Kongjiang Road, Shanghai 200092, P.R. China E-mail: songzhang1665@163.com

Key words: Akt, amniotic fluid-derived mesenchymal stem cells, gene therapy, myocardial ischemia reperfusion injury of Akt-AFMSCs is able to alleviate myocardial I/R injury and improve cardiac function.

\section{Introduction}

Coronary heart disease (CHD) is becoming increasingly prevalent in China. For patients presenting with an acute ST-segment elevation myocardial infarction (STEMI), timely myocardial reperfusion treatment has become a primary strategy. Despite the use of this technique, the morbidity and mortality of patients with STEMI remains problematical. The process of myocardial ischemia-reperfusion (I/R) may, in itself, induce cardiomyocyte death by activating deleterious signaling cascades. There remains no effective therapy for preventing myocardial reperfusion injury. Previously published stem cell therapeutic studies suggested that stem cells may provide a novel approach to protect against I/R injury (1-3). At present, bone marrow mesenchymal stem cells (BM-MSCs) are the most common stem cell for use in transplantation in heart disease. However, the growth potential of BM-MSCs decreases with donor age $(4,5)$, potentially limiting their use in clinical practice. However, age is not a concern with amniotic fluid-derived mesenchymal stem cells (AFMSCs), which are isolated in amniotic fluid. AFMSCs represent a promising alternative in cell-based therapy, given their superior qualities, including ease of acquisition, pluripotency, self-renewal capability, comparatively few ethical concerns, and an absence of teratoma formation $(6,7)$.

However, the survival rate of transplanted cells may be markedly weakened due to an impaired myocardial micro-environment, for example, in the cases of reperfusion injury and inflammation, or in the presence of pro-apoptotic factors (8-10). A low survival rate is a critical problem that needs to be addressed in order to improve the efficiency of stem cell therapy. The activation of Akt, a serine-threonine kinase, maintains stem cells by promoting viability and proliferation $(11,12)$. Furthermore, Akt has been identified as a key molecule for the cell cycling of cardiac myocytes during differentiation, and for the survival/proliferation of progenitor cells (13). Recently, MSCs transduced with Akt have been shown to protect against myocardial injury by enhancing the efficacy of stem cells, inhibiting apoptosis, reducing infarct size and promoting angiogenesis (14-16). 
In the present study, it was hypothesized that the genetic modification of AFMSCs with the pro-survival gene, Akt, would improve the survival of transplanted AFMSCs and enhance their therapeutic efficacy in a rabbit I/R model.

\section{Materials and methods}

Cell culture. AFMSCs were isolated from rabbit amniotic fluid, as previously described (17). AFMSCs were cultured on a $100 \mathrm{~mm}$ diameter-plate $\left(37^{\circ} \mathrm{C}, 5 \% \mathrm{CO}_{2}\right)$ in a growth medium consisting of low glucose Dulbecco's modified Eagle's medium (L-DMEM; Beyotime Institute of Biotechnology, Shanghai, China) supplemented with $20 \%$ fetal bovine serum (FBS; Thermo Fisher Scientific, Waltham, MA, USA) and $1 \%$ penicillin and streptomycin (Beyotime Institute of Biotechnology), and detached from the substrate by incubation in $0.25 \%$ trypsin/EDTA (Beyotime Institute of Biotechnology). The cells were subsequently reseeded at a split ratio of $1: 3$ when growing to $90 \%$ confluence in culture.

Lentiviral transduction of AFMSCs. Rabbit Akt gene was generated by polymerase chain reaction (PCR) using $1 \mu \mathrm{l}$ high-fidelity DNA polymerase (New England Biolabs, Ipswich, MA, USA) and primers containing EcoRI and Xmal sites (forward: 5'-CCGGAATTCGCCACCATG AGCGATGTTACCATTGTGAAAGA-3'; reverse: 5'-TCCC CCCGGGTTATTCCCGTCCACTTGCAGA-3'). The gene fragment and plasmid vector were connected by In-Fusion ${ }^{\circledR}$ technology (Clontech Laboratories, Mountain View, CA, USA) following enzyme digestion, and transformed into competent DH5 $\alpha$ cells. Positive clones containing the lentiviral expression vector were obtained following screening and sequencing. The lentiviral vector was used to transfect 293T cells and package the virus, which led to the determination of the virus titers. AFMSCs at cell passage 3 were transduced with lentivirus vectors in vitro via different values of multiplicity of infection (MOI). The transduction efficiency was obtained according to the optimal MOI, and the expression of the Akt gene was determined using a western blot assay.

Western blot analyses. Protein extracts were obtained from cell lysates of AFMSCs and homogenized myocardium tissue samples by treatment with radioimmunoprecipitation assay lysis buffer (Beyotime Institute of Biotechnology, Shanghai, China). Protein concentrations were determined using a bicinchoninic acid protein assay kit (Beyotime Institute of Biotechnology). Proteins were separated using sodium dodecyl sulfate-polyacrylamide gel electrophoresis (10\% separating gel and 5\% stacking gel; $100 \mathrm{~V}$, blots run for $100 \mathrm{~min}$ ) and transferred on to polyvinylidene difluoride (PVDF) membranes for $90 \mathrm{~min}$ at $250 \mathrm{~mA}$ in Towbin transfer buffer. The PVDF membranes were blocked for $2 \mathrm{~h}$ at room temperature with TBST blocking buffer containing $5 \%$ dry milk and reacted overnight at $4^{\circ} \mathrm{C}$ with the following primary antibodies: Mouse monoclonal anti-Akt antibody (cat. no. 2920; 1:1,000, Cell Signal Technology), mouse monoclonal anti-phosphorylated (P)-Akt antibody (cat. no. 12694; 1:1,000, Cell Signal Technology), mouse monoclonal anti-B-cell lymphoma 2 (Bcl-2) antibody (cat. no. 692; 1:1,000, Abcam, Cambridge, UK), mouse monoclonal anti-connexin 43 antibody (cat. no. 11369; 1:1,500,
Abcam), mouse monoclonal anti-caspase-3 antibody (cat. no. 9668; 1:1,000, Cell Signaling Technology) and mouse monoclonal anti-vascular endothelial growth factor (VEGF) antibody (cat. no. ab1316; 1:1,000, Abcam). After being washed three times, the membranes were treated with goat anti-mouse IgG (cat. no. A0216, Beyotime Institute of Biotechnology; the dilution used was 1:5,000 for the AFMSCs and 1:2,000 for the homogenized myocardium tissue samples). $\beta$-actin and glyceraldehyde-3-phosphate dehydrogenase (GAPDH) served as an internal control for the AFMSCs and myocardium tissue sample, respectively. The enhanced chemiluminescence technique was used for specific protein identification, with Millipore Immobilon Western Chemiluminescent Horseradish Peroxidase substrate (Millipore Corp., Billerica, MA, USA).

5-Bromo-2-deoxyuridine (Brdu) labeling. Once AFMSCs or Akt-AFMSCs had grown to $50 \%$ confluence in culture on a $100 \mathrm{~mm}$ diameter-plate $\left(37^{\circ} \mathrm{C}, 5 \% \mathrm{CO}_{2}\right)$, the culture medium was removed and the cells were incubated with $10 \mu \mathrm{mol} / 1 \mathrm{BrdU}$ (Sigma-Aldrich, St. Louis, MO, USA). At $72 \mathrm{~h}$ after the culture had been initiated, AFMSCs or Akt-AFMSCs were detached with $0.25 \%$ trypsin/EDTA, centrifuged at $22^{\circ} \mathrm{C}$ at $1,000 \mathrm{rpm}$ $(250 \mathrm{~g})$ for $5 \mathrm{~min}$ using a MiniSpin ${ }^{\circledR}$ centrifuge (Eppendorf, Hamburg, Germnay), resuspended at a density of $3 \times 10^{10} / 1$ in $1 \mathrm{ml}$ L-DMEM, and immediately prepared for transplantation.

Myocardial I/R procedure and cell transplantation. All animals were maintained and used as approved by the Animal Experimental Ethics Committee of Xin Hua Hospital Affiliated to Shanghai Jiaotong University School of Medicine. Male New Zealand White rabbits (aged 4 months; 2.4-2.8 kg) were anesthetized with an intravenous injection of sodium pentobarbital (30 mg/kg; Merck \& Co., Inc., Darmstadt, Germany). The chest was opened via mid-sternotomy. A silk thread was passed through the myocardium around a prominent branch of the left anterior descending coronary artery (LAD). Ischemia was induced by pulling the ends of the suture through a segment of a soft tube, which was firmly attached against the artery with a clamp $(18,19)$. The successful induction of ischemia was verified by ST-segment elevation on the electrocardiogram. At 30 min following the ligation, the rabbits were used as a model of myocardial I/R. Prior to the initiation of myocardial reperfusion by releasing the clamp, a suspension of AFMSCs or Akt-AFMSCs $\left(3.0 \times 10^{10} / 1\right.$ in $1 \mathrm{ml}$ L-DMEM; Sigma-Aldrich) was injected into the left ventricular anterior wall at five points.

All the rabbits were randomly divided into four groups ( $n=8$ per group): The sham group (sham), which received the identical surgical procedures as the other groups, excluding the LAD-ligation; the control group (control), which received I/R without cell transplantation; the AFMSC group, which received I/R with transplantation of AFMSCs; and the Akt-AFMSC group, which received I/R with Akt-AFMSC transplantation. The control group without cell therapy received an identical volume of the medium. Functional and histological evaluations were performed 3 weeks following I/R. Rabbits were anesthetized with $3 \%$ sodium pentobarbital, and echocardiography was performed. Subsequently, hearts were quickly excised, cut transversely and processed for morphological, biochemical and molecular studies. 
Detection of donor cells in vivo. For the detection of AFMSCs, specimens were fixed with $4 \%$ paraformaldehyde for $24 \mathrm{~h}$, steeped in 30\% sucrose solution overnight for dehydration, embedded in optimum cutting temperature compound (Sakura Finetek Japan Co., Ltd., Tokyo, Japan). frozen and cut into $5 \mu \mathrm{m}$ sections. Briefly, sections were incubated with mouse monoclonal anti-Brdu antibody (cat. no. B2531; 1:100, Sigma-Aldrich) to detect donor cells. Following a washing step, the sections were incubated with fluorescein isothiocyanate-conjugated goat anti-mouse antibody (cat. no. 115095003; 1:100, (Jackson ImmunoResearch Laboratories, Inc., West Grove, PA, USA). Nuclei were counterstained with 4,6-diamidino-2-phenylindole (DAPI; Beyotime Institute of Biotechnology) after having been washed with PBS. Subsequently, the coverslips were coated with a neutral resin (Ruiqi Biotechnology Co., Ltd., Shanghai, China) for fluorescence microscopic analysis.

Immunohistochemistry. For immunohistochemical staining, heart tissues were fixed in $4 \%$ paraformaldehyde, embedded in paraffin and cut into $5 \mu \mathrm{m}$ sections. The sections were subsequently deparaffinized, rehydrated, and the antigens were retrieved in citrate buffer $(\mathrm{pH}$ 6.0) by boiling in a microwave. The sections were then peroxidase-blocked in 3\% hydrogen peroxide $\left(\mathrm{H}_{2} \mathrm{O}_{2}\right)$ in methanol for $10 \mathrm{~min}$, blocked with 5\% bovine serum albumin in PBS for $30 \mathrm{~min}$, and subsequently incubated with primary antibodies, including mouse monoclonal anti-cardiac troponin T (cTNT) antibody (cat. no. ab10214; 1:100, Abcam) and mouse monoclonal anti-von Willebrand factor (vWF) antibody (cat. no. ab778; $1: 100$, Abcam) at $4^{\circ} \mathrm{C}$ overnight. Following washing in PBS, the sections were incubated with goat anti-mouse secondary antibodies for $1 \mathrm{~h}$ at room temperature. Nuclei were counterstained with hematoxylin (Sigma-Aldrich). Capillary endothelial cells were identified on the basis of immunoreactivity with $\mathrm{vWF}$, and quantified as the number of cells $/ \mathrm{mm}^{2}$. Five fields on the slide were randomly chosen for the counting of stained capillaries at a x200 magnification in a blinded manner. The sections were also stained with hematoxylin-eosin.

Ultrastructural analysis by transmission electron microscopy. The fixed tissues were washed with phosphate buffer $[0.1 \mathrm{M}$, (pH 7.4)] and subsequently post-fixed in identical buffer [0.1 M phosphate buffer ( $\mathrm{pH}$ 7.4)] containing $1 \%$ osmium tetraoxide (Shunbai Biotechnology Co., Ltd., Shanghai, China) at $4^{\circ} \mathrm{C}$ for $2 \mathrm{~h}$. The specimens were subsequently embedded in epoxy-resin (Araldite ${ }^{\circledR}$ CY212; Agar Scientific Ltd., Stansted, UK) to produce tissue blocks and ultrathin sections $(70-80 \mathrm{~nm})$. The sections obtained were stained with uranyl acetate and lead acetate (Shunbai Biotechnology Co., Ltd.), and subsequently examined under a Philips Technai ${ }^{\mathrm{TM}} 10$ transmission election microscope (Phillips, Amsterdam, The Netherlands).

Echocardiography. Rabbit heart function was assessed by transthoracic echocardiography at day 21 following the induction of I/R. All echocardiographic analyses were performed in a blinded manner by the same echocardiographer. The left ventricular internal dimension-diastole (LVIDd) and left ventricular internal dimension-systole (LVIDs) were assessed in short-axis two-dimensional views at the level of the mid-papillary muscle with a GE Vivid 7 system (GE

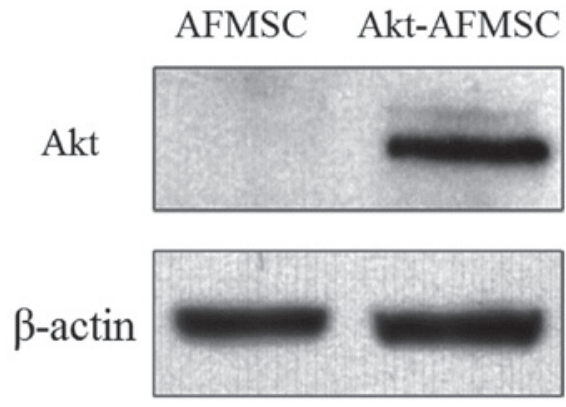

Figure 1. Western blot of rabbit Akt protein.

Healthcare Life Sciences, Shanghai, China) and a standard phased-array. LVIDd and LVIDs were measured from at least three consecutive cardiac cycles. The ejection fraction was calculated as (LVIDd - LVIDs) / LVIDd x 100.

Terminal deoxynucleotidyl-transferase-mediated dUTP nick end labeling (TUNEL) staining. TUNEL assays were performed using an in situ apoptotic cell death detection kit (Roche/Applied Biosystems, Foster City, CA, USA) following the manufacturer's instructions. Sections from each experimental group were examined using a BX53 Olympus microscope (Olympus, Hamburg, Germany). Individual nuclei were visualized at a magnification of $\mathrm{x} 200$ for quantitative analyses. The percentages of apoptotic cells were calculated as the ratio of the number of TUNEL-positive cells to the total number of cells.

Quantitative reverse transcription PCR ( $R T-q P C R)$. The total RNA was extracted, and cDNA was synthesized according to the manufacturer's instructions (Takara Bio, Inc., Otsu, Japan). RT-qPCR was performed using a real-time PCR system (Applied Biosystems ${ }^{\circledR}$ ABI 7500; Thermo Fisher Scientific, Waltham, MA, USA) with the following primers: GATA-4 forward primer, 5'-cagtgagagcettcctcctac-3' and reverse primer, 5'-catagcettgtggggacag-3'; glyceraldehyde-3-phosphate dehydrogenase (GAPDH) forward primer, 5'-atggtgaaggtc ggagtgaa-3' and reverse primer, 5'-tgggtggaatcatactggaac-3'. GAPDH was used as an endogenous control. Relative changes in expression were calculated using the $2^{-\Delta \Delta C q}$ method.

Statistical analysis. Data are expressed as the mean \pm standard error of the mean. Statistical analyses were performed using an unpaired t-test. $\mathrm{P}<0.05$ was considered to indicate a statistically significant value.

\section{Results}

Genetic modification of AFMSCs with the Akt gene. To determine whether stably transfected Akt-AFMSCs exhibited an increased expression of Akt, western blot analysis was performed. A marked increase in the level of Akt in the Akt-AFMSCs compared with the AFMSCs was observed (Fig. 1).

Histological and ultrastructural effects of Akt-AFMSCs on myocardium in $I / R$ rabbits. The sham group exhibited 
A

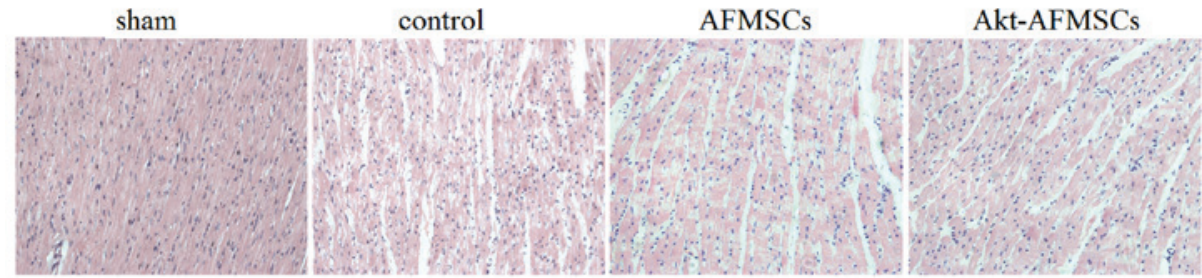

B
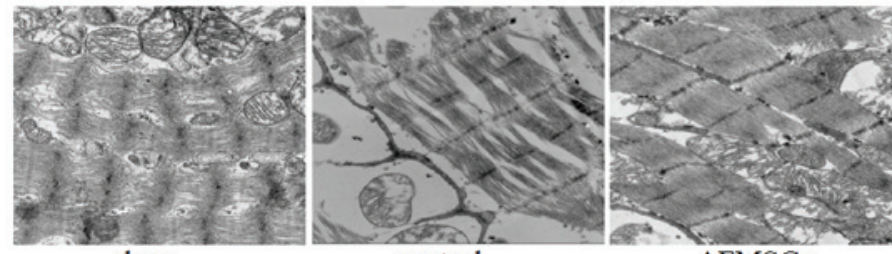

AFMSCs

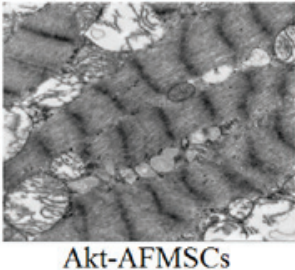

Figure 2. Histological and ultrastructural effects of Akt-AFMSCs on myocardium in I/R rabbits. (A) Histological analysis was performed using hematoxylin-eosin staining. Scale $=100 \mu \mathrm{m}$. (B) Electron microscopic study of myocytes. Scale=500 nm. AFMSCs, amniotic fluid-derived mesenchymal stem cells; $\mathrm{I} / \mathrm{R}$, ischemia-reperfusion.

A

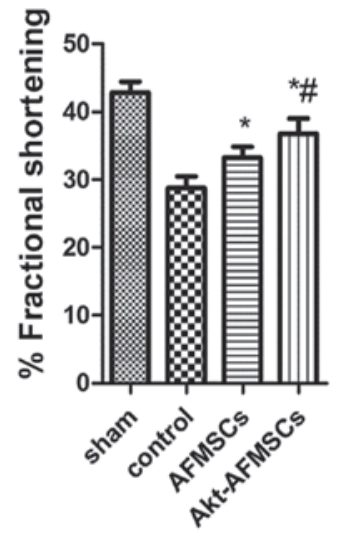

B

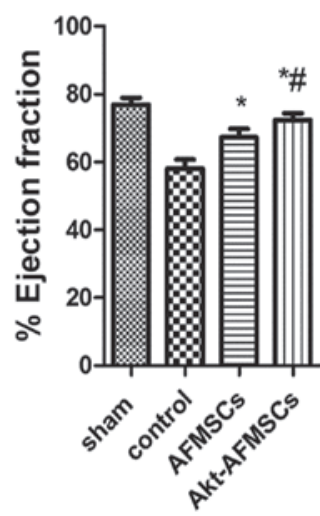

Figure 3. Transplanted Akt-AFMSCs improve cardiac function at day 21 post-ischemia-reperfusion injury. Shown are the (A) fractional shortening $(\%)$ and (B) ejection fractions (\%) for all treatment groups. ${ }^{*} \mathrm{P}<0.05$ vs. control, ${ }^{\#} \mathrm{P}<0.05$ vs. AFMSCs. Data were obtained from $\mathrm{n}=8$ animals/group. AFMSCs, amniotic fluid-derived mesenchymal stem cells.

normal myocardium structure, whereas the control group showed significant membrane damage of cardiomyocytes, with inflammatory cell infiltration and myonecrosis. The AFMSC and Akt-AFMSC groups revealed significant structural improvements (Fig. 2A). Compared with the sham group, observations made under the transmission electron microscope demonstrated that the control group were subjected to significant disorganization of chromatin condensation, swollen and disrupted cristae in the mitochondria and a loss of cytoplasmic vacuoles and myofibers (Fig. 2B). However, there was a modest alleviation of ultrastructural damage in hearts transplanted with AFMSCs or Akt-AFMSCs.

Echocardiography in Akt-AFMSCs transplanted in an $I / R$ model. Echocardiography was performed to assess the effects of transplanted Akt-AFMSCs on cardiac function 3 weeks following $\mathrm{I} / \mathrm{R}$. As shown in Fig. $3 \mathrm{~A}$ and $\mathrm{B}, \mathrm{I} / \mathrm{R}$ injury significantly decreased fractional shortening and the ejection fraction in the rabbit heart $(\mathrm{P}<0.05)$. Compared with the control group, the AFMSC and Akt-AFMSC groups exhib-

$\mathbf{A}$

DAPI
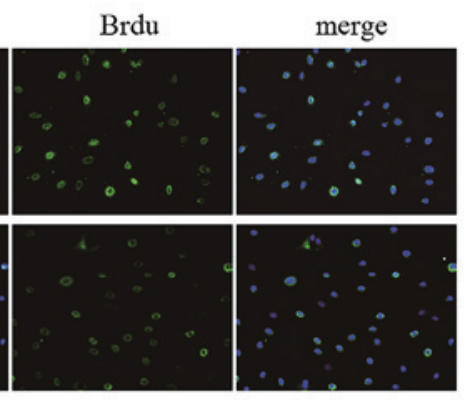

B

AFMSCs
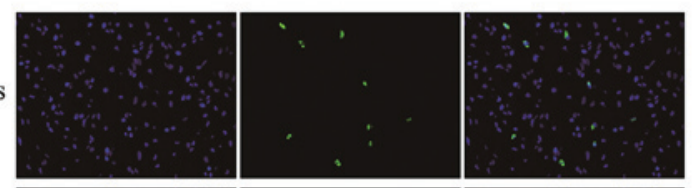

Akt-AFMSCs
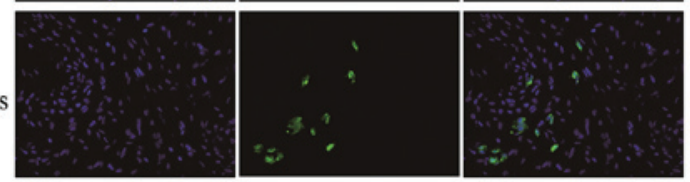

Figure 4. Tracking of AFMSCs in vitro and in vivo. (A) Fluorescence microscopic study of Brdu-labeled AFMSCs and Akt-AFMSCs in vitro. Brdu-labeled AFMSCs and Akt-AFMSCs were stained with anti-Brdu (green) and counterstained with DAPI for nuclear visualization (blue). Magnification, x200. (B) Fluorescence microscopy of Brdu-labeled AFMSCs and Akt-AFMSCs at day 21 after the transplantation of AFMSCs or Akt-AFMSCs in heart tissue with ischemia-reperfusion injury. Magnification, x400. DAPI, 4,6-diamidino-2-phenylindole; AFMSCs, amniotic fluid-derived mesenchymal stem cells; Brdu, 5-bromo-2-deoxyuridine.

ited higher fractional shortening $(29.18 \pm 2.36$ vs. $33.65 \pm 2.81$ and $36.89 \pm 3.02 \%, \mathrm{P}<0.05$, respectively) and ejection fractions $(58.62 \pm 3.47$ vs. $67.42 \pm 3.03$ and $72.02 \pm 2.89, \mathrm{P}<0.05$, respectively), suggesting that Akt-AFMSCs exhibit an improved level of cardiac function against I/R injury.

Tracking of AFMSCs in vitro and in vivo. In our cell culture system, Brdu was used to label AFMSCs for subsequent cell tracking. Following treatment with Brdu at $10 \mu \mathrm{M}$ for $72 \mathrm{~h}$, the percentages of AFMSCs and Akt-AFMSCs labeled with Brdu were $90 \pm 2.8$ and $89 \pm 3.5 \%$, respectively 

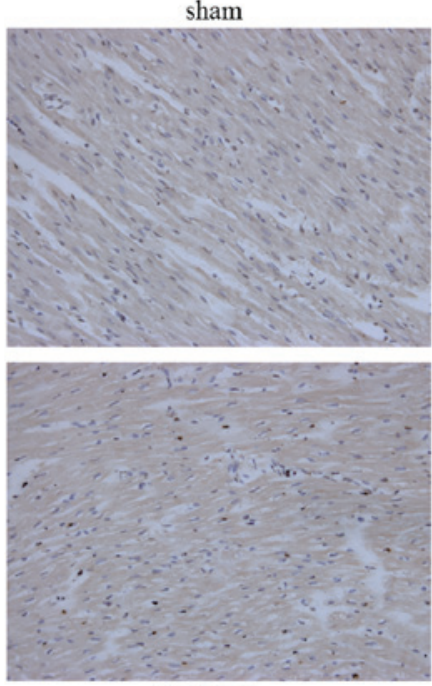

AFMSCs
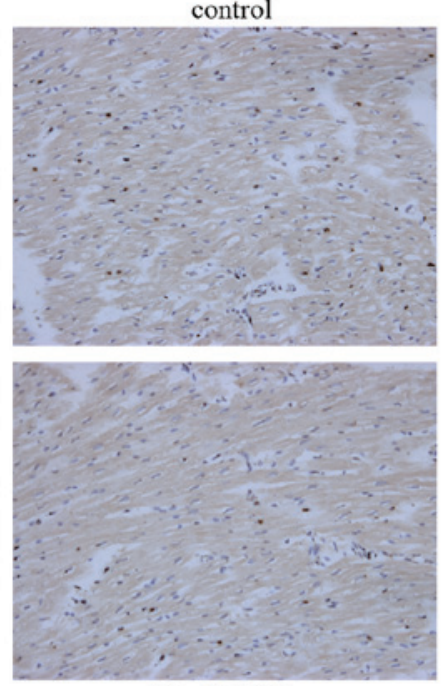

Akt-AFMSCs
B

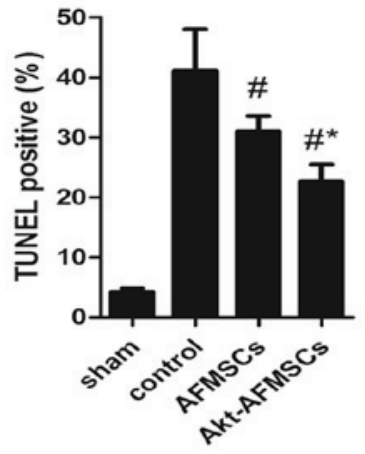

Figure 5. Apoptosis quantified in myocardial sections by TUNEL staining. (A) Representative photomicrographs of TUNEL staining. In the images, apoptotic cells are characterized by nuclear condensation, and are stained dark brown under a light microscope. Magnification, x200. (B) Quantitative analysis of apoptotic nuclei per tissue section is presented as the mean \pm standard deviation. ${ }^{~} \mathrm{P}<0.05$ vs. control; ${ }^{~} \mathrm{P}<0.05$ vs. AFMSCs. AFMSCs, amniotic fluid-derived mesenchymal stem cells; TUNEL, terminal deoxynucleotidyl-transferase-mediated dUTP nick end labeling.

A

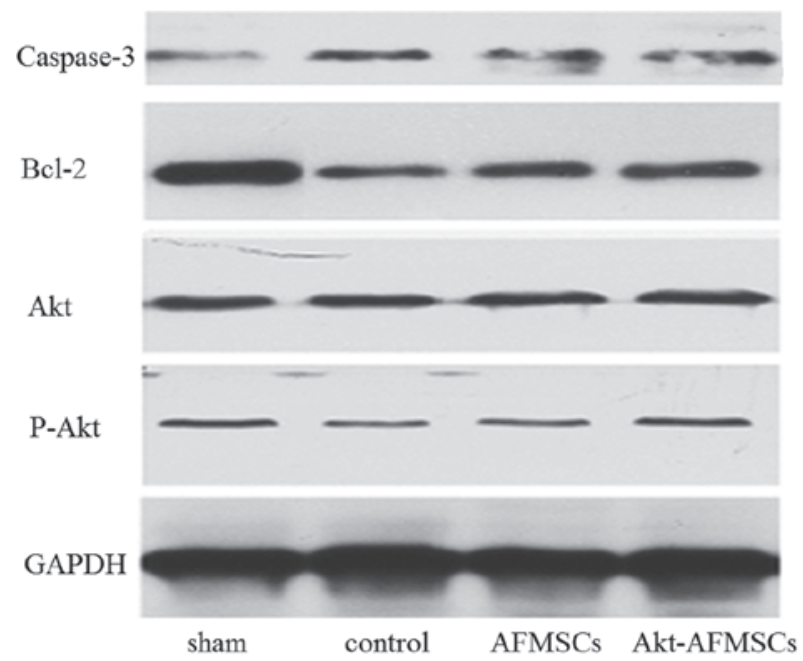

B

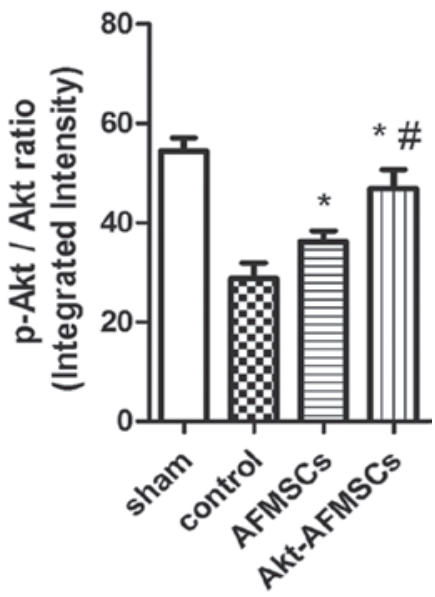

C

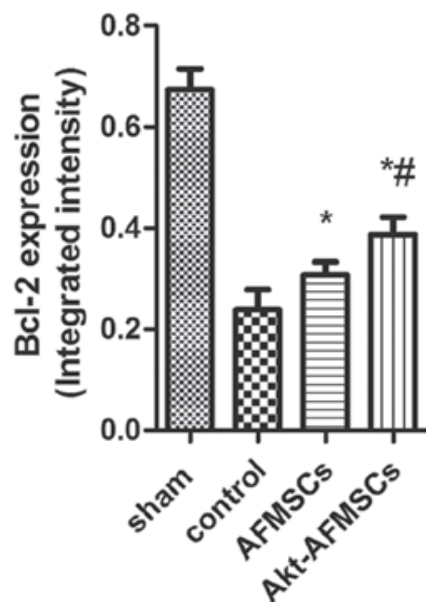

D

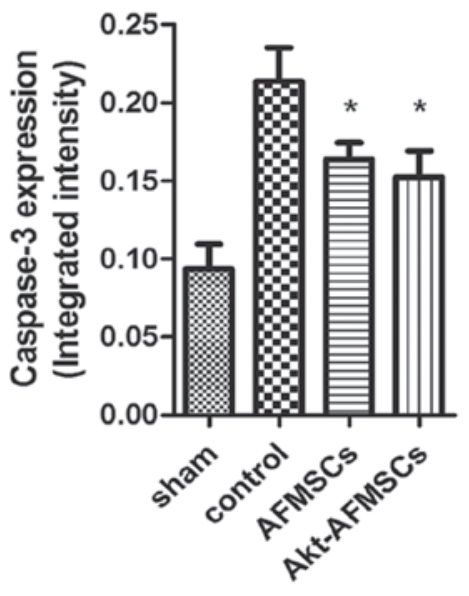

Figure 6. (A) Protein expression of caspase-3, Bcl-2, Akt and P-Akt in hearts was assessed by western blotting. The Akt-AFMSC group showed an increased phosphorylation of (B) Akt (B) and (C) Bcl-2 in the left ventricle of rabbits subjected to myocardial ischemia-reperfusion. (D) The Akt-AFMSC and AFMSC groups showed a decreased level of caspase-3 in the left ventricle of rabbit subjected to myocardial ischemia-reperfusion. Data obtained from semiquantitative densitometry are presented as the mean \pm standard deviation for eight rabbits per group. ${ }^{*} \mathrm{P}<0.05$ vs. control; ${ }^{*} \mathrm{P}<0.05$ vs. AFMSCs. Bcl-2, B-cell lymphoma 2 ; P, phosphorylated; AFMSCs, amniotic fluid-derived mesenchymal stem cells. 
A

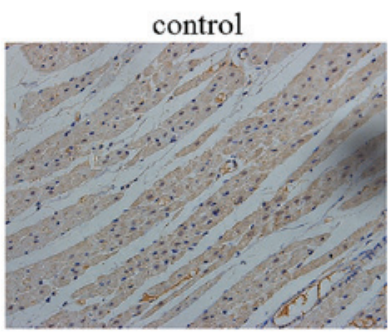

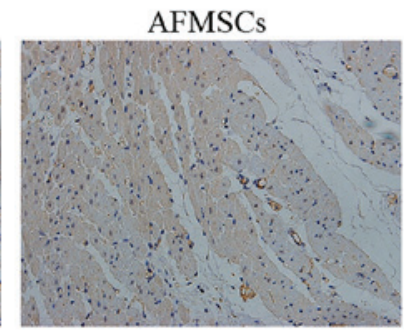

B

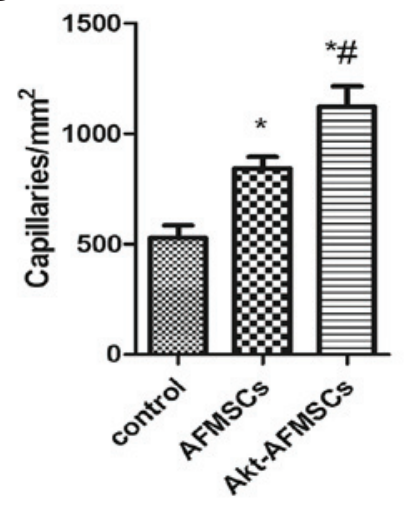

$\mathbf{C}$

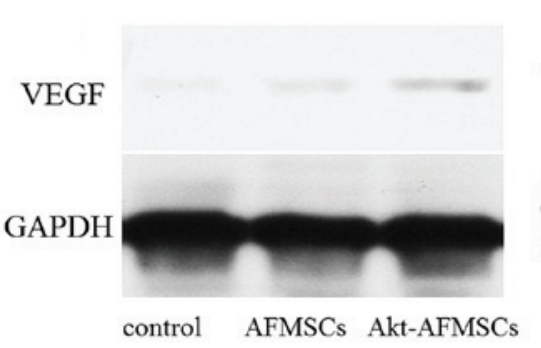

Akt-AFMSCs

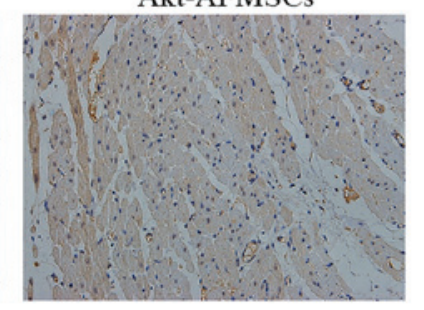

D

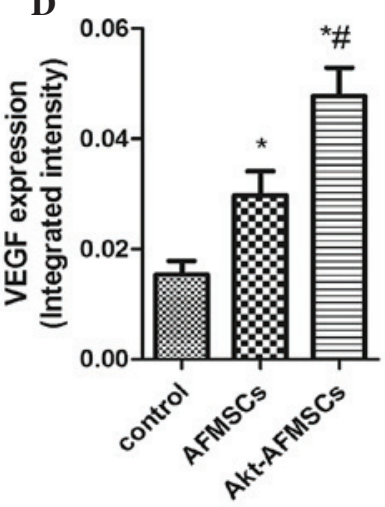

Figure 7. (A) Capillary density was measured using anti-von Willebrand factor immunohistology 21 days after transplantation. Blue staining shows the nuclei; brown staining shows the capillaries. Magnification, x200. (B) The capillary density in the AFMSC and Akt-AFMSC groups was significantly higher than in the control group. ${ }^{*} \mathrm{P}<0.05$ vs. control; ${ }^{~} \mathrm{P}<0.05$ vs. AFMSCs. (C) VEGF secretion in recipient hearts was assessed by western blotting. (D) The levels of VEGF in the Akt-AFMSC group was significantly higher compared with that in control group. " $\mathrm{P}<0.05$ vs. control; " $\mathrm{P}<0.05 \mathrm{vs}$. AFMSCs. GAPDH, glyceraldehyde-3-phosphate dehydrogenase; VEGF, vascular endothelial growth factor; AFMSCs, amniotic fluid-derived mesenchymal stem cells.

(Fig. 4A). Immunohistochemical staining of Brdu in tissues was performed in order to track the cells in the myocardium. As shown in Fig. 4B, a greater number of cells remained in the Akt-AFMSC group compared with the AFMSC group. $(\mathrm{P}<0.05)$. The average number of Brdu-labeled Akt-AFMSCs and AFMSCs was 14.8 $\pm 3.0 /$ high-power field and 9.3 $\pm 2.6 /$ high-power field, respectively.

Cardiomyocyte apoptosis following Akt-AFMSC transplantation. The number of TUNEL-positive cardiomyocytes was significantly lower $(\mathrm{P}<0.05)$ in hearts transplanted with AFMSCs and Akt-AFMSCs (Fig. 5A). Notably, the number of apoptotic muclei was markedly reduced in the Akt-AFMSCs group compared with the AFMSCs group (Fig. 5B), suggesting that Akt is a potential pro-survival factor that inhibits I/R-induced cardiomyocyte apoptosis. To examine whether P-Akt, which can inhibit protein synthesis in apoptosis, is implicated in the cardioprotection of Akt-overexpressing AFMSCs in transplantation, western blot analysis was performed for Akt and P-Akt (Fig. 6A). These data suggest that hearts transplanted with either Akt-AFMSCs or AFMSCs exhibited a marked increase in P-Akt expression compared with the controls. Furthermore, transplantation of the Akt-AFMSCs increased the expression of P-Akt compared with the AFMSC group (Fig. 6B). However, no statistical significances attributable to Akt were observed in all the groups.

Multiple signaling pathways contribute to apoptosis, including caspase-3 and Bcl-2. Western blot analysis was performed for $\mathrm{Bcl}-2$ and caspase-3 (Fig. 6A). Bcl-2 exerts a major role in determining the death or survival of cells after the application of apoptotic stimuli. Fig. 6C shows how the Bcl-2 level was decreased in the control group, and this decrease was partly reversed by the transplantation of Akt-AFMSCs or AFMSCs. Caspase-3 is an important component of the final pathway leading to the cell apoptosis, and myocardial caspase-3 activity is considered as a marker of I/R-induced cardiomyocyte apoptosis. As shown in Fig. 6D, myocardial $\mathrm{I} / \mathrm{R}$ resulted in a noticeable increase in caspase-3 compared with the sham group. A significant decrease in caspase-3 was observed in the Akt-AFMSC and AFMSC groups compared with the control group. However, no statistically significant differences were observed in the caspase-3 levels between the Akt-AFMSC and AFMSC groups.

Angiogenesis in Akt-AFMSCs transplantation. Immunohistological analysis revealed that the capillary density was significantly higher in the Akt-AFMSC and AFMSC groups compared with the control group. Notably, in the Akt-AFMSC group, the increased level of angiogenesis was greater compared with the AFMSC group (Fig. 7A and B). To examine whether Akt-AFMSCs exerted any effect on secretion of VEGF in the heart, western blot analysis was performed for VEGF in myocardium tissue (Fig. 7C). The expression of VEGF in the zone of I/R injury in the AFMSC group was significantly higher compared with the control group, and also higher in the Akt-AFMSC group compared with the AFMSC group (Fig. 7D), suggesting that genetic modification of AFMSCs with the Akt gene may further promote the release of VEGF. 
A

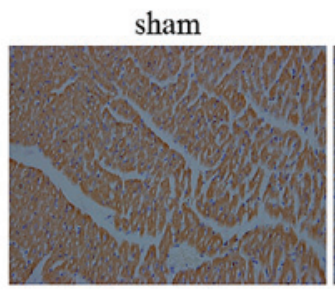

B

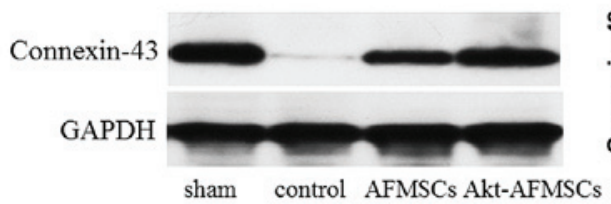

control

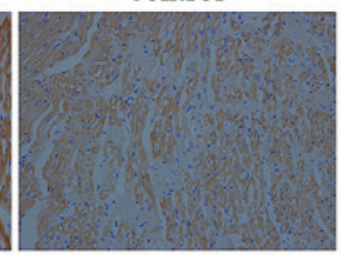

AFMSCs

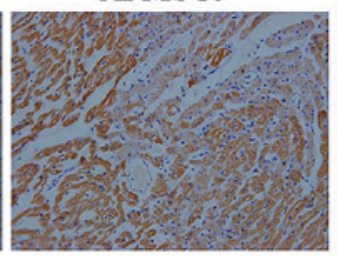

Akt-AFMSCs

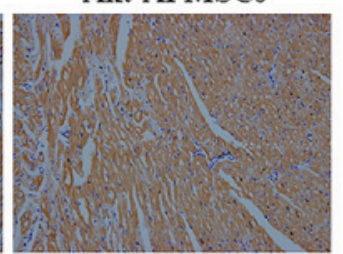

C

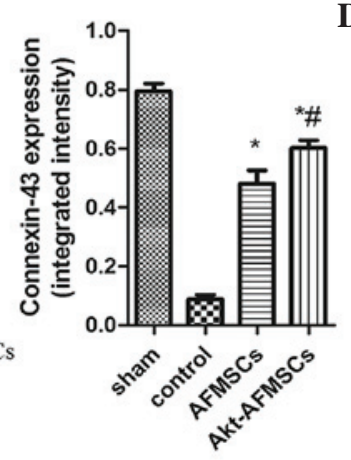

D

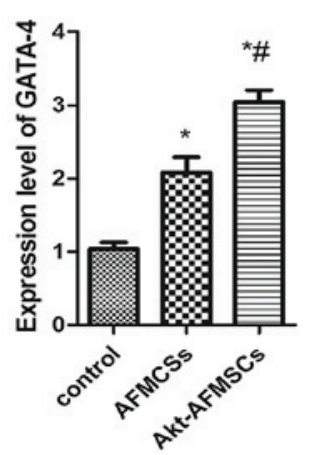

Figure 8. Expression of GATA-4, connexin 43 and cTNT in transplanted Akt-AFMSCs. (A) Representative photomicrographs of tissue sections stained for cTNT at day 21 post-ischemia-reperfusion injury cTNT staining was identified as being increased in the Akt-AFMSCs by brown staining. Magnification, x200. (B) The connexin-43 level was assessed by western blot analysis. (C) Protein expression of connexin-43 by western blotting in the Akt-AFMSC group was the highest compared with the control and AFMSC groups. ${ }^{*} \mathrm{P}<0.05$ vs. control; ${ }^{\#} \mathrm{P}<0.05$ vs. AFMSCs. (D) Quantitative real-time polymerase chain reaction revealed that the gene expression of GATA-4 was enhanced in the Akt-AFMSC group at 21 days after cell transplantation ( $\mathrm{n}=8$ animals per group). ${ }^{*} \mathrm{P}<0.05$ vs. control, ${ }^{\text {}} \mathrm{P}<0.05$ vs. AFMSCs. GAPDH, glyceraldehyde-3-phosphate dehydrogenase; cTNT, cardiac troponin T; AFMSCs, amniotic fluid-derived mesenchymal stem cells.

Expression of GATA-4, connexin 43 and cTNT in the transplanted Akt-AFMSCs. Immunohistological analysis revealed that the expression of cTNT was markedly higher in the Akt-AFMSC and AFMSC groups compared with the control group (Fig. 8A). The Akt-AFMSC group also revealed a greater increase in the expression of cTNT compared with the AFMSC group. The Akt-AFMSC group also exhibited a higher expression of the cardiac myocyte-specific markers, GATA-4, connexin 43 and cTNT, suggesting that the transplantation of Akt-AFMSCs may promote cardiac regeneration in myocardial I/R injury. At 21 days following myocardial I/R, the difference in protein expression of connexin-43, as determined by western blotting, in the Akt-AFMSC, control and AFMSC groups reached its peak (Fig. 8B and C). Compared with the control group, the Akt-AFMSC and AFMSCs groups revealed higher levels of gene expression of GATA-4 $(1.00 \pm 0.24$ vs. $3.08 \pm 0.23$ and $2.11 \pm 0.19, \mathrm{P}<0.05$, respectively) (Fig. 8D).

\section{Discussion}

For patients with a STEMI, the opportunity to intervene in acute myocardial ischemia is limited to the time of myocardial reperfusion. The effects of myocardial reperfusion by percutaneous coronary intervention continues to improve with earlier times of reperfusion. However, the process of myocardial I/R may itself induce cardiomyocyte death by activating deleterious signaling cascades. Previously, a number of emerging therapeutic strategies for preventing lethal myocardial reperfusion injury have shown promise in small clinical studies. These strategies include ischemic post-conditioning, remote ischemic preconditioning, therapeutic hyperoxemia and hypothermia, pharmacological agents, including adenosine, atrial natriuretic peptide, atorvastatin, erythropoietin, exenatide, delcasertib, glucose-insulin-potassium (GIK), cyclosporin A, sodium nitrite and TRO40303. However, certain interventions have produced disappointing results, whereas the effects of others are now being investigated through multicenter and randomized clinical trials (20). There remains no effective therapeutic agent for preventing myocardial reperfusion injury.

Previous reports suggested that myocardial I/R injury may be weakened through the transplantation of stem cells, such as embryonic stem cells (ESCs) and adult stem cells (ASCs) (21). These reports prompted our assessment of whether AFMSC myocardial transplantation confers protection of the heart against I/R injury. Based on the source of the stem cells, cells are divided into ESCs and ASCs. As a novel category of stem cell, AFMSCs have several advantages over other stem cells. For instance, considered to be at an intermediate stage between ESCs and ASCs, AFMSCs express the two types of stem cell markers, proliferate rapidly, and differentiate into cells of all three embryonic germ layers $(22,23)$. Unlike ESCs, AFMSCs do not form teratomas when injected into immune-deficient mice, and the isolation of AFMSCs does not have ethical implications. ASCs are isolated from the most differentiated adult tissues (24). Compared with ASCs, the isolation of AFMSCs is a simpler process, and large numbers of AFMSCs may be isolated from amniotic fluid, proliferate rapidly, and do not require a supportive feeder layer $(17,25)$. In addition, ASCs are few in the adult organism, and their number decreases with age (26). Furthermore, recently, a technique for acquiring pluripotent cells from differentiated cells of an adult organism 
through genetic modification, which are termed induced pluripotent stem cells (iPS cells), was established. However, the use of iPS cells for cell therapy is limited by the tumorigenicity of these cells. As such, AFMSCs are an attractive cell source for applications in regenerative medicine due to their high proliferative capacity, immunomodulatory activity, multipotency and the lack of significant immunogenicity.

AFMSC-based research on regenerative medicine applications is increasingly becoming more prevalent. For differentiation purposes, AFMSCs have been force-induced into a number of lineages, including those of liver, cartilage, bone, pancreas, muscle, neuronal and endothelial lineages in vitro (27). In addition to multipotency, AFMSCs have the ability to secrete cytokines, many of which are pro-regenerative, pro-angiogenic or immunomodulatory. Previous animal experiments have shown that AFMSCs may be useful in the treatment of renal failure (28), skin wounds (29), fulminant hepatic failure (30), diabetes mellitus (31), and other condtions. Additionally, a burgeoning number of studies have shown that AFMSCs may be differentiated into endothelial and cardiomyogenic lineages $(6,32)$, and implantation of AFMSCs into cardiac tissue following myocardial infarction can be beneficial $(33,34)$.

More attention has been paid to I/R following the re-establishment of the coronary blood flow following myocardial infarction. Therapeutic strategies on cardiac protection against I/R injury are urgently needed. Studies of pre- and post-conditioning have shown that $\mathrm{I} / \mathrm{R}$ injury is able to initiate several pro-survival kinase cascades, including the phosphoinositide 3-kinase/Akt signaling pathway (35). Previous studies, for example, Mangi et al (15), have also shown that Akt-overexpressing MSCs present more benefits against I/R injury (15). Akt, a serine-threonine kinase, maintains stem cells by promoting viability and proliferation. In addition, Akt is an important factor in the regulation of intercellular glycometabolism that increases energy support during hypoxia. However, its role in the genetic modification of AFMSCs in protecting against myocardial I/R injury has yet to be elucidated. Therefore, the present study was designed to determine whether overexpressing Akt in AFMSCs would alleviate I/R injury. The results have demonstrated that transplanted AFMSCs (especially AFMSCs overexpressing Akt) significantly improve pathological myocardial I/R injury, cardiac function, cardiomyocyte apoptosis, angiogenesis and the expression of GATA-4, connexin 43 and cTNT. These results were similar to those obtained from a majority of stem cell transplantations against myocardial I/R injury, indicating that AFMSCs may be more suitable as a cell source for applications in stem cell therapy due to their advantage over ESCs and ASCs.

The present study has shown that the Akt-AFMSC group had more stem cell retention compared with the AFMSC group. This finding is explained, in part, by Akt gene modifications, which enhanced the viability of stem cells and resistance to apoptosis. It has also been demonstrated that Akt is an excellent therapeutic gene for preserving MSC viability in the early post-transplantation period (15).

To date, it is generally agreed that the mechanism of cardiovascular regeneration by stem cells is mediated by the release of a myriad of cytokines and chemokines. In terms of
AFMSCs, a previous study demonstrated that these cells are able to secrete a number of growth factors at concentrations higher than those of MSCs (29). In addition, Akt-modified MSCs are able to excrete additional factors, including VEGF, fibroblast growth factor-2, hepatocyte growth factor (HGF), insulin growth factor (IGF)-1 and thymosin $\beta$-4 (36). In the present study, the promotion of angiogenesis by AFMSCs was accompanied by a higher expression of VEGF in cardiac tissue. A decrease in cardiomyocyte apoptosis was accompanied by decreased levels of caspase-3 and elevated levels of P-Akt and Bcl-2, suggesting that the Akt-AFMSC transplantation group resulted in a greater benefit compared with the other groups. Taken together, our results are consistent with the hypothesis that Akt-overexpressing AFMSCs provide a cardioprotective effect via a paracrine mechanism.

With regard to higher expression levels of GATA-4, connexin 43 and cTNT following Akt-AFMSC transplantation, the effects may be a consequence of the above-mentioned decrease in cardiomyocyte apoptosis in the Akt-AFMSC group, and/or certain cytokines excreted by Akt-AFMSCs, which are able to enhance the migration and activation of resident cardiac stem cells, leading to differentiation into cardiomyocytes. As for the latter mechanism, it has been well documented that IGF-1/HGF are able to activate endogenous cardiac stem cells (37). AFMSCs have also been reported to excrete HGF (29).

In conclusion, the present study suggests that AFMSCs and Akt-AFMSCs protect the heart against I/R injury in rabbits. These results were similar to those for stem cell transplantation against myocardial I/R injury, indicating that AFMSCs may be more suitable as a stem cell source for application in stem cell therapies due to their advantages over ESCs and ASCs. The protective effect may be due to delivery of secreted cytokines, promotion of neoangiogenesis, prevention of cardiomyocyte apoptosis, transdifferentiation into cardiomyocytes, or a combination of these effects. Genetically, modification through Akt appears to represent an improved therapeutic approach, promoting the viability of transplanted AFMSCs, and thereby enhancing their protective effects against cardiac I/R injury.

\section{Acknowledgements}

This work was supported by a Nature Science Foundation of China Grant (no. 81170124/H0203).

\section{References}

1. Angoulvant D, Ivanes F, Ferrera R, Matthews PG, Nataf S and Ovize M: Mesenchymal stem cell conditioned media attenuates in vitro and ex vivo myocardial reperfusion injury. J Heart Lung Transplant 30: 95-102, 2011.

2. Preda MB, Rønningen T, Burlacu A, Simionescu M, Moskaug JØ and Valen G: Remote transplantation of mesenchymal stem cells protects the heart against ischemia-reperfusion injury. Stem Cells 32: 2123-2134, 2014.

3. Wang Y, Abarbanell AM, Herrmann JL, Weil BR, Manukyan MC, Poynter JA and Meldrum DR: TLR4 inhibits mesenchymal stem cell (MSC) STAT3 activation and thereby exerts deleterious effects on MSC-mediated cardioprotection. PloS One 5: e14206, 2010.

4. Dexheimer V, Mueller S, Braatz F and Richter W: Reduced reactivation from dormancy but maintained lineage choice of human mesenchymal stem cells with donor age. PloS One 6: e22980, 2011. 
5. Kanawa M, Igarashi A, Ronald VS, Higashi Y, Kurihara H, Sugiyama M, Saskianti T, Pan H and Kato Y: Age-dependent decrease in the chondrogenic potential of human bone marrow mesenchymal stromal cells expanded with fibroblast growth factor-2. Cytotherapy 15: 1062-1072, 2013.

6. Maioli M, Contini G, Santaniello S, Bandiera P, Pigliaru G, Sanna R, Rinaldi S, Delitala AP, Montella A, Bagella L and Ventura C: Amniotic fluid stem cells morph into a cardiovascular lineage: Analysis of a chemically induced cardiac and vascular commitment. Drug Des Devel Ther 7: 1063-1073, 2013.

7. Rennie K, Gruslin A, Hengstschläger M, Pei D, Cai J, Nikaido T and Bani-Yaghoub M: Applications of amniotic membrane and fluid in stem cell biology and regenerative medicine. Stem Cells Int 2012: 721538, 2012.

8. Cheng AS and Yau TM: Paracrine effects of cell transplantation: Strategies to augment the efficacy of cell therapies Semin Thorac Cardiovasc Surg 20: 94-101, 2008.

9. Zhang M, Methot D, Poppa V, Fujio Y, Walsh K and Murry CE: Cardiomyocyte grafting for cardiac repair: Graft cell death and anti-death strategies. J Mol Cell Cardiol 33: 907-921, 2001.

10. Balsam LB, Wagers AJ, Christensen JL, Kofidis T, Weissman IL and Robbins RC: Haematopoietic stem cells adopt mature haematopoietic fates in ischaemic myocardium. Nature 428 : 668-673, 2004.

11. Hammerman PS, Fox CJ, Birnbaum MJ and Thompson CB: Pim and Akt oncogenes are independent regulators of hematopoietic cell growth and survival. Blood 105: 4477-4483, 2005.

12. Kim SJ, Cheon SH, Yoo SJ, Kwon J, Park JH, Kim CG, Rhee K, You S, Lee JY, Roh SI and Yoon HS: Contribution of the PI3K/Akt/PKB signal pathway to maintenance of self-renewal in human embryonic stem cells. FEBS Lett 579: 534-540, 2005

13. Catalucci D and Condorelli G: Effects of Akt on cardiac myocytes: Location counts. Circ Res 99: 339-341, 2006.

14. Lim SY, Kim YS, Ahn Y, Jeong MH, Hong MH, Joo SY, Nam KI, Cho JG, Kang PM and Park JC: The effects of mesenchymal stem cells transduced with Akt in a porcine myocardial infarction model. Cardiovasc Res 70: 530-542, 2006.

15. Mangi AA, Noiseux N, Kong D, He H, Rezvani M, Ingwall JS and Dzau VJ: Mesenchymal stem cells modified with Akt prevent remodeling and restore performance of infarcted hearts. Nat Med 9: 1195-1201, 2003.

16. Yu YS, Shen ZY, Ye WX, Huang HY, Hua F, Chen YH, Chen K, Lao WJ and Tao L: AKT-modified autologous intracoronary mesenchymal stem cells prevent remodeling and repair in swine infarcted myocardium. Chin Med J (Engl) 123: 1702-1708, 2010.

17. Fei X, Jiang S, Zhang S, Li Y, Ge J,He B, Goldstein S and Ruiz G: Isolation, culture and identification of amniotic fluid-derived mesenchymal stem cells. Cell Biochem Biophys 67: 689-694, 2013.

18. Zhang S, Zhang M, Goldstein S, Li Y, Ge J, He B and Ruiz G: The effect of c-fos on acute myocardial infarction and the significance of metoprolol intervention in a rat model. Cell Biochem Biophys 65: 249-255, 2013.

19. Zhang S, Liu X, Goldstein S, Li Y, Ge J, He B, Fei X, Wang Z and Ruiz G: Role of the JAK/STAT signaling pathway in the pathogenesis of acute myocardial infarction in rats and its effect on NF- $\kappa$ B expression. Mol Med Rep 7: 93-98, 2013.

20. Chen C, Xu Y and Song Y: IGF-1 gene-modified muscle-derived stem cells are resistant to oxidative stress via enhanced activation of IGF-1R/PI3K/AKT signaling and secretion of VEGF Mol Cell Biochem 386: 167-175, 2014.

21. Takashima S, Tempel D and Duckers HJ: Current outlook of cardiac stem cell therapy towards a clinical application. Heart 99: 1772-1784, 2013.
22. Kunisaki SM, Fuchs JR, Steigman SA and Fauza DO: A comparative analysis of cartilage engineered from different perinatal mesenchymal progenitor cells. Tissue Eng 13: 2633-2644, 2007

23. Kolambkar YM, Peister A, Soker S, Atala A and Guldberg RE: Chondrogenic differentiation of amniotic fluid-derived stem cells. J Mol Histol 38: 405-413, 2007.

24. Lanfranchi A, Porta F and Chirico G: Stem cells and the frontiers of neonatology. Early Hum Dev 85 (Suppl 10): S15-S18, 2009.

25. Davydova DA: Stem cells in human amniotic fluid. Izv Akad Nauk Ser Biol: 517-526, 2010 (In Russian).

26. Sessarego N, Parodi A, Podestà M, Benvenuto F, Mogni M, Raviolo V, Lituania M, Kunkl A, Ferlazzo G, Bricarelli FD, et al: Multipotent mesenchymal stromal cells from amniotic fluid: Solid perspectives for clinical application. Haematologica 93: 339-346, 2008.

27. Zhou J, Wang D, Liang T, Guo Q and Zhang G: Amniotic fluid-derived mesenchymal stem cells: Characteristics and therapeutic applications. Arch Gynecol Obstet 290: 223-231, 2014.

28. Baulier E, Favreau F, Le Corf A, Jayle C, Schneider F, Goujon JM, Feraud O, Bennaceur-Griscelli A, Hauet T and Turhan AG: Amniotic fluid-derived mesenchymal stem cells prevent fibrosis and preserve renal function in a preclinical porcine model of kidney transplantation. Stem Cells Transl Med 3: 809-820, 2014.

29. Skardal A, Mack D, Kapetanovic E, Atala A, Jackson JD, Yoo J and Soker S: Bioprinted amniotic fluid-derived stem cells accelerate healing of large skin wounds. Stem Cells Transl Med 1: 792-802, 2012

30. Zheng YB, Zhang XH, Huang ZL, Lin CS, Lai J, Gu YR, Lin BL, Xie DY, Xie SB, Peng L and Gao ZL: Amniotic-fluid-derived mesenchymal stem cells overexpressing interleukin-1 receptor antagonist improve fulminant hepatic failure. PloS One 7: e41392, 2012

31. Villani V, Milanesi A, Sedrakyan S, Da Sacco S, Angelow S, Conconi MT, Di Liddo R, De Filippo R and Perin L: Amniotic fluid stem cells prevent $\beta$-cell injury. Cytotherapy 16: 41-55, 2014.

32. Yeh YC, Wei HJ, Lee WY, Yu CL, Chang Y, Hsu LW, Chung MF, Tsai MS, Hwang SM and Sung HW: Cellular cardiomyoplasty with human amniotic fluid stem cells: In vitro and in vivo studies. Tissue Eng Part A 16: 1925-1936, 2010.

33. Bollini S, Pozzobon M, Nobles M, Riegler J, Dong X, Piccoli M, Chiavegato A, Price AN, Ghionzoli M, Cheung KK, et al: In vitro and in vivo cardiomyogenic differentiation of amniotic fluid stem cells. Stem Cell Rev 7: 364-380, 2011.

34. Lee WY, Wei HJ, Lin WW, Yeh YC, Hwang SM, Wang JJ, Tsai MS, Chang Y and Sung HW: Enhancement of cell retention and functional benefits in myocardial infarction using human amniotic-fluid stem-cell bodies enriched with endogenous ECM. Biomaterials 32: 5558-5567, 2011.

35. Murphy E and Steenbergen C: Mechanisms underlying acute protection from cardiac ischemia-reperfusion injury. Physiol Rev 88: 581-609, 2008.

36. Gnecchi M, He H, Noiseux N, Liang OD, Zhang L, Morello F, Mu H, Melo LG, Pratt RE, Ingwall JS and Dzau VJ: Evidence supporting paracrine hypothesis for Akt-modified mesenchymal stem cell-mediated cardiac protection and functional improvement. FASEB J 20: 661-669, 2006.

37. Ellison GM, Torella D, Dellegrottaglie S, Perez-Martinez C, Perez de Prado A, Vicinanza C, Purushothaman S, Galuppo V, Iaconetti C, Waring CD, et al: Endogenous cardiac stem cell activation by insulin-like growth factor-1/hepatocyte growth factor intracoronary injection fosters survival and regeneration of the infarcted pig heart. J Am Coll Cardiol 58: 977-986, 2011 